\title{
New additions of basidiomycetous fungi in Indian mycoflora
}

\section{Kumari $\mathrm{B}^{1}$ and Atri NS ${ }^{2}$}

\author{
${ }^{1}$ Abhilashi Institute of Life Sciences, Tanda, Nerchock Mandi (H.P.) \\ ${ }^{2}$ Department of Botany, Punjabi University, Patiala 147 002, India \\ Corresponding author, E-Mail: babita.thkr@gmail.com
}

Kumari B, Atri NS 2013 - New additions of basidiomycetous fungi in Indian mycoflora. Mycosphere 4(1), 53-59, Doi 10.5943 /mycosphere/4/1/4

Three new Leucoagaricus taxa were found in various localities of North West India. Leucoagaricus albidus sp. nov., Leucoagaricus barsii var. bulbobasilarus var. nov., and Leucoagaricus tener var. brevisporus var. nov. are described and illustrated.

Key words - Agaricaeae - fleshy fungi - India - Leucoagaricus - taxonomy

\section{Article Information}

Received 21 November 2012

Accepted 10 December 2012

Published online 28 January 2013

*Corresponding author: Kumari B - e-mail - babita.thkr@gmail.com

\section{Introduction}

The genus Leucoagaricus Locq. ex Singer (family Agaricaceae) is characterized by small to medium sized basidiocarps with lepiotoid to pluteoid habitat; radially fibrillose to floccose, scaly and rarely granulose pileus having very short striate margin which may be collariate lamellae; central stipe with membranous, annulus, metachromatic basidiospores, absence of clamp connections, presence of cheilocystidia and absence of pleurocystidia. It differs from closely related Leucocoprinus in having very shortly pectinate pileus margin instead of long pectinate margin. Initially it was treated as a subgenus of Leucocoprinus by Locquin (1943), but elevated from subgenus level to generic level by Singer (1948). Over 90 species are known (Kirk et al. 2008), although only 8 species are recorded from India (Leucoagaricus crystallifer Vellinga, L. fuligineus Pegler, L. holosericeus (Gillet) M.M. Moser, L. excoriatus (Schaeff.)
Singer, L. melanotrichus var. melanotrichus (Malençon \& Bertault) Trimbach, $L$. rubrotinctus (Peck.) Singer, L. rhodocephalus (Berk.) Pegler and L. sublittoralis (Kühner ex Hora) Singer (Bilgrami et al., 1979; Sarbhoy et al., 1992; Jamalludin et al., 2001 and Natarajan et al., 2005). During surveys to various localities of North India a new species, Leucoagaricus albidus sp. nov., and two new varieties, L. barssii var. bulbobasilarus var. nov. and $L$. tener var. brevisporus var. nov. were found and are described in this paper.

\section{Materials and methods}

Standard methods for collection, preservation and description of agarics were followed, using the terminology of Atri et al. (2005). Colour notations in the macroscopical descriptions are from Kornerup \& Wanscher (1978). The specimens were hot air dried and preserved in cellophane bags containing 1-4 dichlorobenzene. Macroscopic examination 
was carried out on fresh specimens in the field. Microscopic characters were studied from freehand sections mounted in $5 \% \mathrm{KOH}$, stained of sterigmata. The spore shape quotient $(\mathrm{Q}+$ $\mathrm{L} / \mathrm{W}$ ) was calculated considering the mean value of length and width of 20 basidiospores. The specimens have been deposited in the Herbarium of Punjabi University Patiala (Punjab) India. Microscopic details are shown in Figs 1-3.

\section{Taxonomic observations}

Leucoagaricus albidus B. Kumari \& N.S. Atri sp. nov.

MycoBank no.: MB 802380

Fig. $1 \mathrm{~A}-\mathrm{F}$

Etymology - The name of the species is based on shining whitish carpophores.

Fructifications $3.0-7.0 \mathrm{~cm}$ in height. Pileus $2.0-3.0 \mathrm{~cm}$ in diameter, convex to hemispherical, surface shining, white to yellowish white (4A2), brownish orange on drying; margin regular, incurved, not splitting at maturity; cuticle fully peeling; flesh $0.5 \mathrm{~cm}$ thick, white, changing to yellowish white to pinkish on bruising; taste and odour mild. Pileal veil in the form of appendiculate veil fragments. Lamellae free, unequal, of two lengths, subdistant to crowded, $0.5 \mathrm{~cm}$ broad, yellowish white in young specimens, reddish white (8A2) to brown at maturity; gill edges lacerate. Stipe central, $2.0-6.3 \mathrm{~cm}$ long, 0.6$1.1 \mathrm{~cm}$ broad, pale yellow (4A3), obclavate, concolorous with the pileus, hollow, scaly, glabrous; annulate, annulus single, superior, ring like.

Basidiospores $\quad 5.0-6.7(-7.6) \times 3.4-$ $4.8(-5) \mu \mathrm{m}(\mathrm{Q}=1.4)$, broadly ellipsoid to amygdaliform with rounded apex, thickwalled, congophilous, cyanophilous, dextrinoid, weakly metachromatic in cresyl blue, apical pore absent. Basidia 19.3-29 $\times$ 6.4-9.6 $\mu \mathrm{m}$, clavate, tetrasporic; sterigmata 1.6-2.4 $\mu \mathrm{m}$ long. Pleurocystidia 22.5-32.2 $\times$ 8.8-12.8 $\mu \mathrm{m}$, scattered, clavate to pyriform; gill edges heteromorphous. Cheilocystidia 19.3-30.6 × 7.2-16.0 $\mu \mathrm{m}$, crowded, broadly clavate to pyriform, granular, scattered, hyaline.

Pileus cuticle a trichoderm, consisting of regular turf of branched, septate cylindrical with $1 \%$ Congo red. Microscopic line drawings were made with the aid of a camera lucida at 1000x. Basidium length excludes the length to tubular tipped elements measuring 4.0-8.0 $\mu \mathrm{m}$ in width, some of these with intracellular pigmentation; context homoiomerous; hymenophoral trama regular, tramal hyphae up to $10 \mu \mathrm{m}$ in width; subhymenium pseudoparenchymatous, well developed. Pileal veil elements septate, granular, up to $9.8 \mu \mathrm{m}$ in width. Stipe surface hyphae parallel 4.0-26.0 $\mu \mathrm{m}$ in width. All hyphae lack clamp connections.

Collection examined - Himachal Pradesh, Mandi, Sarkaghat, Bakarta, $(850 \mathrm{~m})$, growing scattered or in caespitose clusters in rice field, Babita Kumari, 12 July 2009, PUN 4309 (Holotype).

Remarks - It is an interesting whitish coloured lepiotoid mushroom, in which flesh turns light yellowish to pinkish when bruised. In its outward morphology it appears quite similar to L. leucothites (Vittad.) Wasser of which Vellinga (2001) recognized one more variant, namely var. carneifolinus beside var. typica. The presently examined fungus/species is quite close to L. leucothites var. typica from which it differs in possessing smaller aporus basidiospores than 5 7.5-11.0 $\times 5.0-7.0 \mu \mathrm{m}$ (Vellinga 2001). In view of the presence of shining whitish carpophores with flesh discolouring yellow to pinkish on bruising, annulate stipe, smaller spores and presence of pleurocystidia, a new species has been proposed to accommodate this collection.

Leucoagaricus barssii var. bulbobasilarus B. Kumari \& N.S. Atri var. nov.

MycoBank 802381

Fig. $2 \mathrm{~A}-\mathrm{F}$

Etymology - The name of the variety is based on the bulbous base of stipe.

Fructifications $3.4-6.5 \mathrm{~cm}$ in height. Pileus $2.5-5.6 \mathrm{~cm}$ in diameter, plano-convex; surface pale yellow (3A2) covered with greyish brown (5C2) scales, squamules more concentrated towards the center; margin irregular, splitting at maturity; cuticle fully peeling; flesh up to $0.4 \mathrm{~cm}$ thick, off white, unchanging; taste mild, odour fruity. Lamellae free, collariate, unequal, of 3-4 lengths, subdistant, pale yellow (3A3), $0.6 \mathrm{~cm}$ broad; 

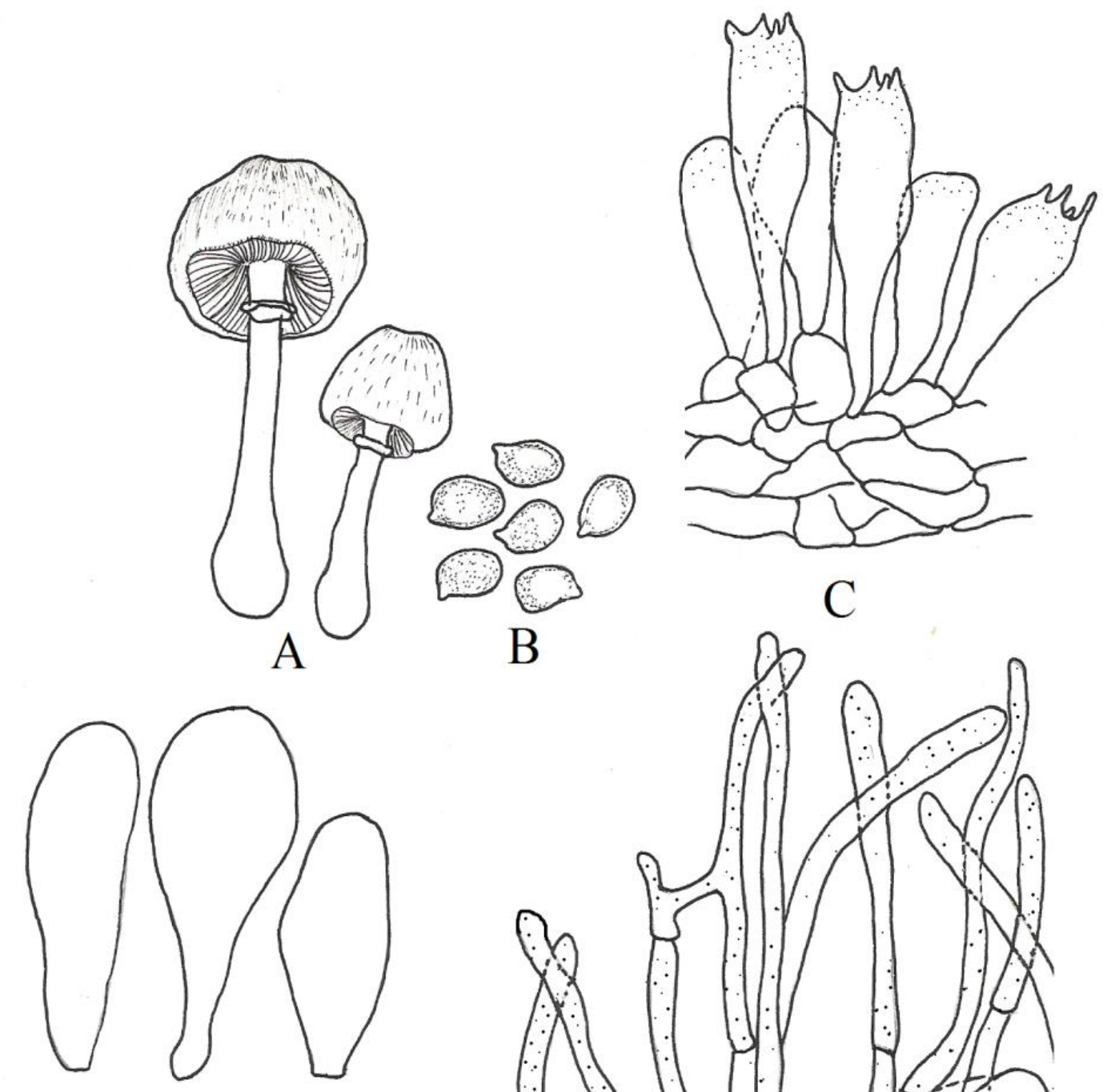

$\mathrm{D}$

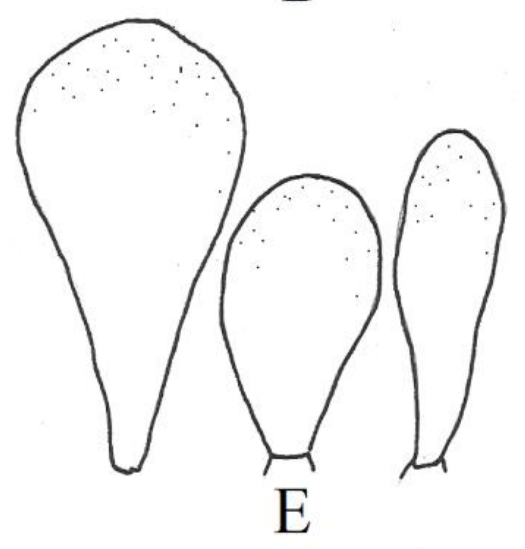

$$
\text { B-E } \frac{\mathrm{A} \frac{3 \mathrm{~cm}}{20 \mu \mathrm{m}}}{\mathrm{F} \stackrel{20 \mu \mathrm{m}}{ }}
$$

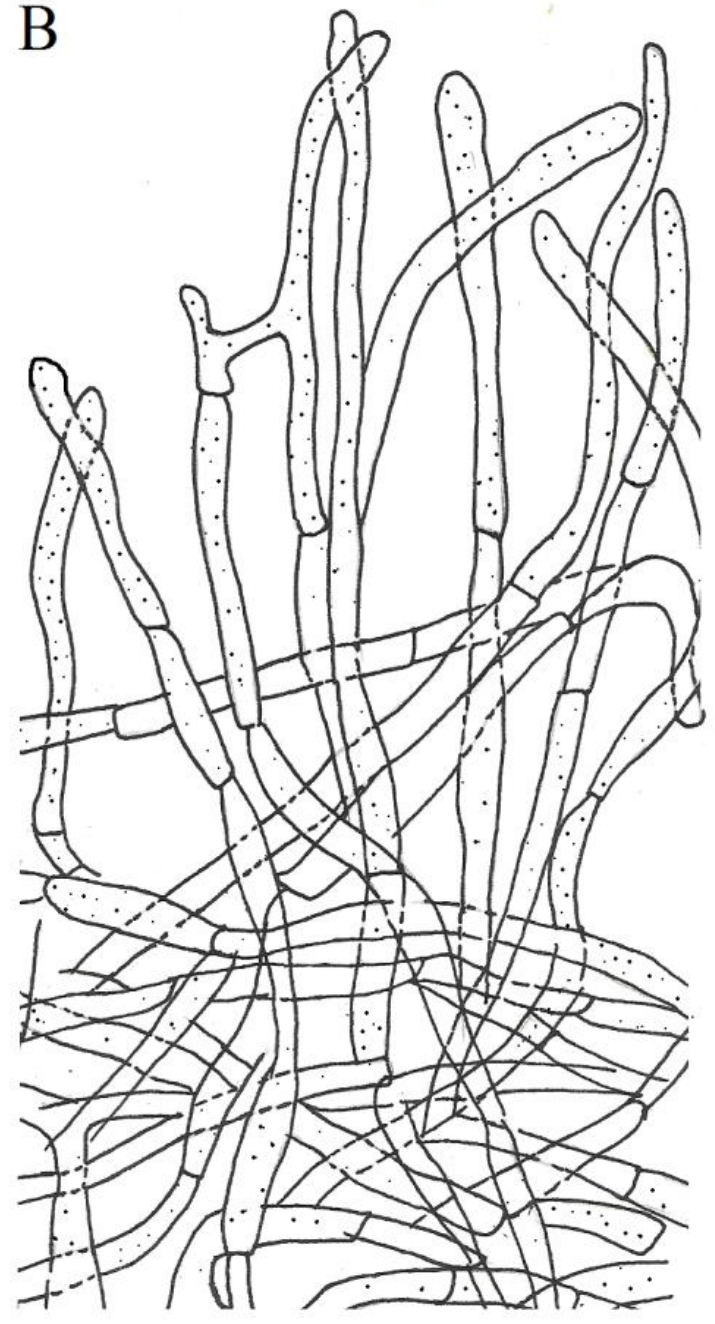

$\mathrm{F}$

Fig. 1 A-F - Leucoagaricus albidus sp. nov.: A Carpophores. B Basidiospores. C Hymenophore with Basidia. D Pleurocystidia. E Cheilocystidia. F Pileus trichodermial elements. 

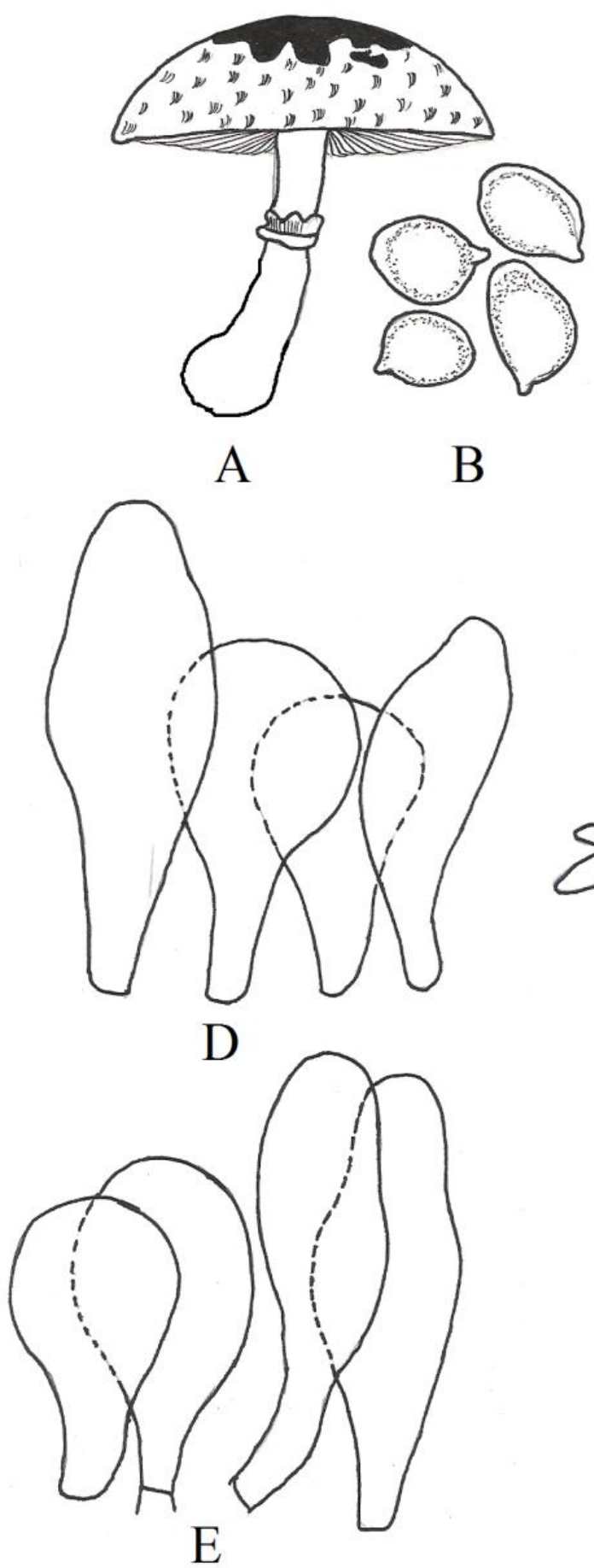

$$
\text { B-E } \frac{\mathrm{A} \frac{3 \mathrm{~cm}}{20 \mu \mathrm{m}}}{\mathrm{F} \stackrel{20 \mu \mathrm{m}}{\longrightarrow}}
$$
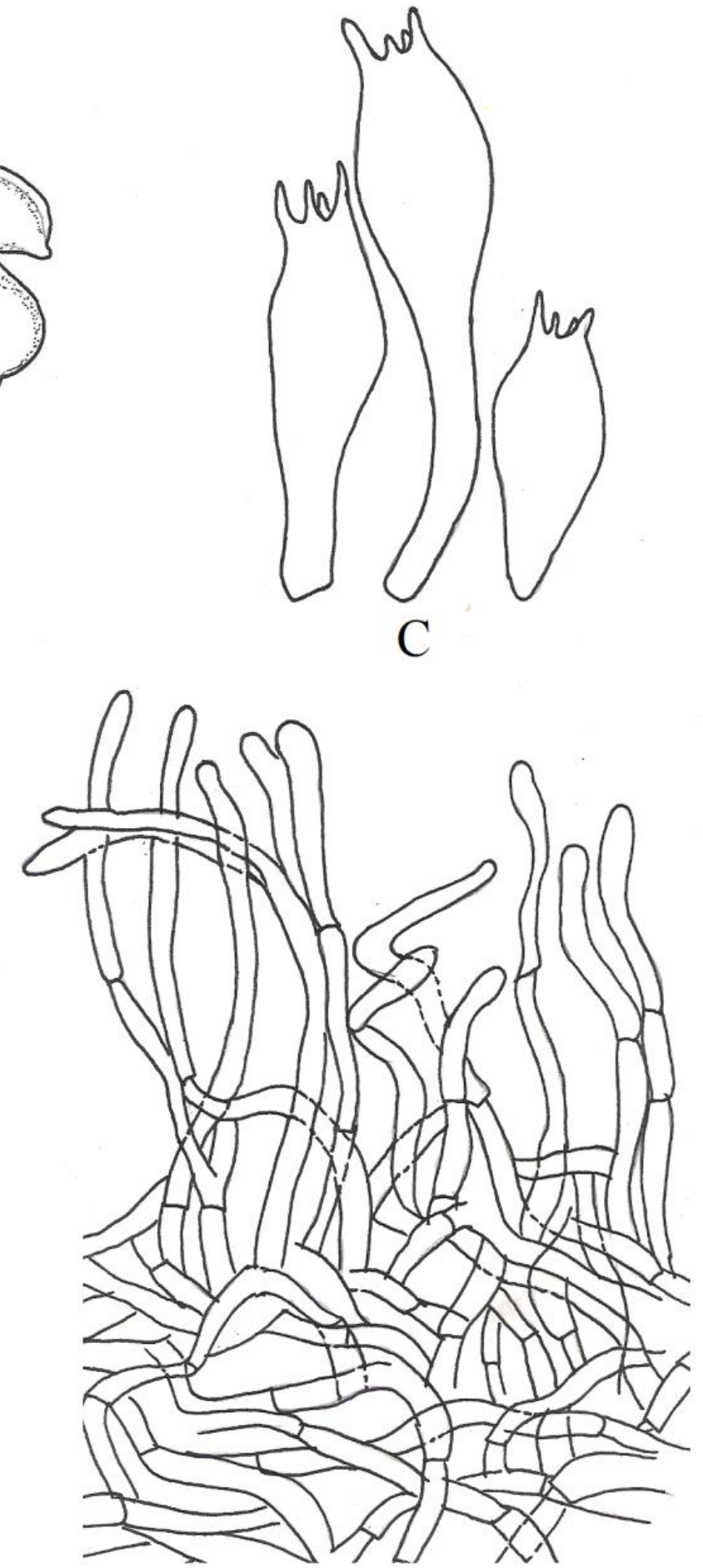

Fig. 2 A-F - Leucoagaricus barsii var. bulbobasilarus var. nov.: A Carpophore. B Basidiospores. C Basidia. D Cheilocystidia. E Pleurocystidia. F Pileus cuticle elements. 
gill edges fimbriate; spore deposit pale yellow (4A3). Stipe central, $5.8 \mathrm{~cm}$ long, $1.1 \mathrm{~cm}$ broad, yellowish white (4A2), brownish on bruising, obclavate with bulbous base, hollow; annulus single, thick, attached.

Basidiospores 8.0-10.0 × 6.4-7.3 $\mu \mathrm{m}$ $(\mathrm{Q}=1.2)$, broadly ellipsoid with rounded apex, inamyloid, congophilous, cyanophilous, dextrinoid, weakly metachromatic in cresyl blue, apical pore absent. Basidia 22.5-48.3 × 9.6-12 $\mu \mathrm{m}$, broadly clavate, tetrasporic; sterigmata 3.2-4.8 $\mu \mathrm{m}$ long; gill edges heteromorphous, largely sterile with occasional basidial elements. Cheilocystidia 25.8-42.0 $\times$ 9.6-16 $\mu \mathrm{m}$, crowded, broadly clavate to pyriform, thick-walled, hyaline. Pleurocystidia 23.6-41.5 × 9-15.5 $\mu \mathrm{m}$, present near the gill edges which are similar to cheilocystidia.

Pileus cuticle a disrupted epicutis of radial hyphae measuring $4.0-8.0 \mu \mathrm{m}$ in width; context homoiomerous, well developed; gill trama subregular, tramal hyphae $3.2-4.8 \mu \mathrm{m}$ in width; subhymenium pseudoparenchymatous. Stipe surface hyphae parallel running, 4.0-8.0 $\mu \mathrm{m}$ in width. Clamp connections present in the basal mycelium.

Collection examined - Patiala, Girls Hostel road, Punjabi University Patiala (250 $\mathrm{m})$, growing scattered on sandy soil, Babita Kumari, 18 June 2008, PUN 4311 (Holotype). Remarks - This is an interesting mushroom growing solitary on sandy soil. The species is easily recognized by greyish fibrillose brown squamules on the pileus surface, presence of both cheilocystidia and pleurocystidia. In many of its features it is closely related to $L$. macrorhizus Locq. ex Singer, which mainly differs from it in having a big carpophore with brownish pileus and large spores with germ pore (Vellinga 2001). Because of the presence of pleurocystidia, it can also be compared with L. americanus (Peck) Vellinga. On the basis of large spore size and bulbous base L. barssii var. bulbobasilarus has been proposed. $L$. barssii (Zeller) Vellinga is not documented earlier from India.

Leucoagaricus tener var. brevisporus B. Kumari \& N.S. Atri var. nov. MycoBank 802382
Etymology - The name of the variety is based on the small brown spores.

Fructifications $7.5-8 \mathrm{~cm}$ high. Pileus $2.2-5.0 \mathrm{~cm}$ wide, convex to campanulate, disc brown (6D7), greyish orange (5B5) to ochraceous towards the margin with greyish orange (5B5) appressed fibrillose scales on a pale yellow (4A3) background; scales more dense towards the center in young sporophores; margin irregular, splitting at maturity; cuticle fully peeling; flesh $0.3-0.4 \mathrm{~cm}$ thick, off white, unchanging; taste mild and odour sour (like lemon). Lamellae free, close to crowded, unequal, of 3-4 lengths, yellowish white (4A2), unchanging; gill edges smooth; spore deposit yellowish white (4A2). Stipe 7.0-7.6 $\mathrm{cm}$ long, $0.5-0.6 \mathrm{~cm}$ broad, yellowish white (4A2); yellowish brown on bruising, concolorous with the pileus, expanding slightly at the base, hollow, pruinose fibrillose; annulus single, funnel shaped with brown dots on the lower side, presence of water drops on the upper portion of stipe surface.

Basidiospores 4.8-6.5 × 3.2-4.0 $\mu \mathrm{m}(\mathrm{Q}$ $=1.56$ ), ellipsoid to slightly amygdaliform, pseudoamyloid, guttulate with single guttule, metachromatic in cresyl blue, apical pore absent. Basidia 12.9-20.9 × 5.6-7.24 $\mu \mathrm{m}$, thin walled, hyaline, tetrasporic; sterigmata 2.4-3.2 $\mu \mathrm{m}$ long; gill edges sterile. Cheilocystidia $19.3-40.3 \times 5.6-12.8 \mu \mathrm{m}$, crowded, versiform, varying from clavate to lageniform or even irregular with lateral apical prolongations. Pleurocystidia absent.

Pileus cuticle formed of more or less irregular trichoderm consisting of short cylindrical, thin-walled segments measuring $20.0-78.0 \mu \mathrm{m}$ in length and $4.0-8.0 \mu \mathrm{m}$ in width, devoid of clamp connections at slightly constricted septa and readily dissociating towards the terminal portion, terminal elements clavate to cylindrical with obtuse tips; context homoiomerous; gill trama subregular; subhymenium pseudoparenchymatous well developed, formed of more or less isodiametric elements. Stipe cuticle hyphae parallel, 4.0 $12.0 \mu \mathrm{m}$ in width with some projecting branched elements measuring $6.0-8.0 \mu \mathrm{m}$ in width. Clamp connections absent throughout. 

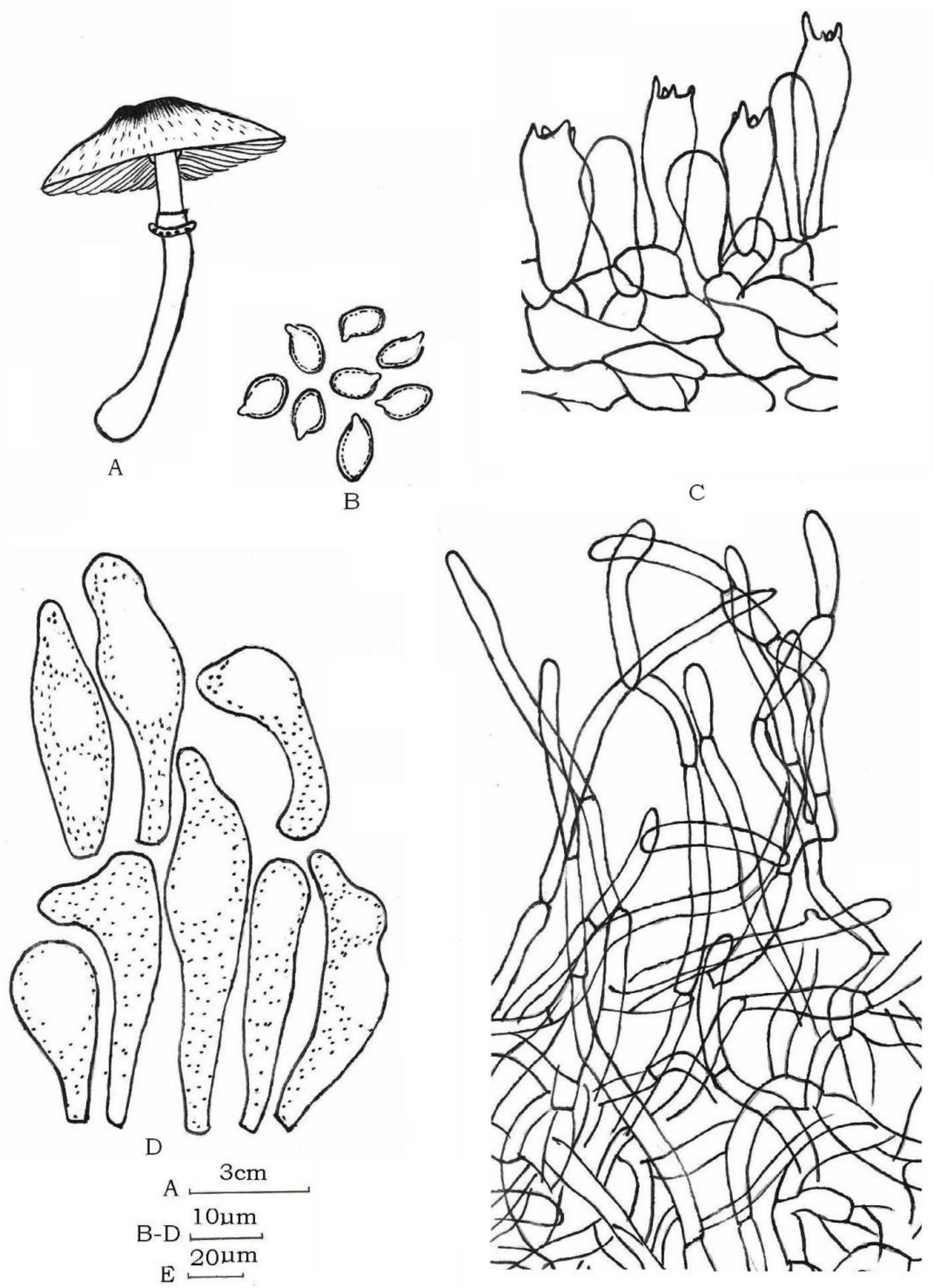

$\mathrm{E}$

Fig. 3 A-E - Leucoagaricus tener var. brevisporus var. nov.: A Carpophore. B Basidiospores. C Basidia. D Cheilocystidia. E Pileus trichodermial elements. 
Collection examined - Himachal Pradesh, Mandi, Sarkaghat, Bakarata $(850 \mathrm{~m})$, growing scattered on soil in association with roots of Bamboo, Babita Kumari, 5 August 2009, PUN 3939 (Holotype).

Remarks - This variety can be easily identified in the field by its brownish disc with greyish orange fibrillose flecks on a pale yellow background and presence of water drops on the fresh carpophores, especially on the upper portion of stipe. The macroscopic and microscopic features of this mushroom are in close agreement with L. tener (P.D. Orton) Bon. It is a small spored variant of Lepiota tener with spores measuring 4.8-6.5 × 3.2-4.0 $\mu \mathrm{m}$ compared to $6.0-8.0 \times 3.2-4.0 \mu \mathrm{m}$ for the type variety (Reid 1995).

\section{Acknowledgements}

Authors acknowledge the grant-in-aid under SAP-III program by the University Grants Commission to the Department of Botany, Punjabi University, Patiala of which Mycology and Plant Pathology is one of the thrust area.

\section{References}

Atri NS, Kaur A, Kaur H. 2005 - Wild Mushrooms - Collection and Identification. Frontiers in Mushroom Biotechnology. (Rai RD, Upadhyay RC, Sharma SR eds). NRCM Chambaghat, Solan, pp. 9-26.

Bilgrami KS, Jamaluddin, Rizwi AM. 1979 Fungi of India. Part-I: Lists and References. Today and Tomorrow's Printers and Publishers, New Delhi.
Jamalludin, Goswami MG, Ojha BM. 2001 Fungi of India (1989-2001). Forest Pathology Division. Tropical Forest Research Institute, Jabalpur (MP) Pawan Kumar, Scientific Publishers, India.

Kirk PM, Cannon PF, Minter DW, Stalpers JA (eds). 2008 - Dictionary of the Fungi, $10^{\text {th }}$ edn. CABI Publishing, UK.

Kornerup A, Wanscher JH. 1978 - Methuen Handbook of Colour, $3^{\text {rd }}$ edn. Eyre Methuen, London.

Locquin M. 1943 - Etude de development des spores du genre Leucocoprinus Pat. Bulletin Mensuel de la Société Linnéenne de Lyon. 12, 92.

Natarajan K, Kumaresan V, Narayanan K. 2005 - A checklist of Indian agarics and boletes (1984-2002). Kavaka. 33, 92.

Reid DA. 1995 - Observations on Leucoagaricus melanotrichus and similar species. Mycotaxon. 80: 325-336.

Sarbhoy AK, Varshney JL, Agarwal DK. 1992 - Fungi of India. CBS Publishers and Distributors. 4596/1-4, 11, Daryaganj, New Delhi.

Singer R. 1948. New and interesting species of Basidiomycetes. Michigan Academy of Science, Arts \& Letters. 32: 103-150.

Vellinga EC. 2001 - Macrolepiota Sing., Leucocoprinus Pat., Leucoagaricus (Locq. ex) Sing., Lepiota (Pers.: Fr.) SF Gray, Cystolepiota Sing., Melanophyllum Velen. - In: Noordeloos, M.E., Kuyper, T.W. and Vellinga, E.C. eds. Flora Agaricina Neerlandica, vol. 5, 64-162, Rotterdam. 